\title{
Occurrence of Astringent Oligomeric Proanthocyanidins in Legume Seeds*
}

\author{
Toshiaki Ariga, Yasuo Asao, Hiroshi Sugimoto \\ and Tamotsu YokotsuKA** \\ Central Research Laboratories, Kikkoman Corporation, \\ Noda-shi, Chiba 278, Japan
}

Received December 4, 1980

\begin{abstract}
The distribution of oligomeric proanthocyanidins in legume seeds was investigated in connection with organoleptic astringency. The seeds contained various kinds of oligomers (dimers hexamers). The total oligomer contents were at least $0.040 \%$ in azuki beans, $0.017 \%$ in black soybeans, $0.005 \%$ in mung beans and a trace amount in regular soybeans. Each aliquot of aqueous solution of partially purified proanthocyanidin oligomers tasted astringent even at a concentration of $0.001 \%(\mathrm{w} / \mathrm{v})$. The possible contribution of oligomeric proanthocyanidins and polymeric proanthocyanidins to the astringent off-taste of legume seeds is discussed.
\end{abstract}

Proteins in legume seeds have not yet been utilized partly because of their astringent offtaste. No satisfactory explanation for this offtaste, however, has been given and no effective technique for removing the organoleptic astringency from legume seeds has been developed until now.

On the other hand, several investigations on astringent components in fruits and their products, such as avocado fruits and applies, have been carried out during the last twenty years. Geissman and Dittmar ${ }^{1)}$ revealed that dimeric proanthocyanidins isolated from avocado fruits tasted astringent. $\mathrm{Lea}^{2}$ reported that higher-molecular-weight procyanidins contributed to the astringency of cider.

Recently, some workers reported the occurrence of condensed tannins in some legume seeds. Martin-Tanguy et al. $^{3)}$ reported that horse bean seed-coats contain proanthocyanidin-type tannins. Price et al $^{4}{ }^{4}$ measured the condensed flavan contents of some legume seeds from the view point of their nutritional effects. Until now, however, there has been no paper reporting the occurrence of

* Studies on Astringent Components in Legume Seeds. Part I.

** Production Division, Kikkoman Corporation. oligomeric proanthocyanidins in legume seeds. And no report has described the contribution of oligomeric and polymeric proanthocyanidins to the astringent off-taste of legume seeds.

This paper describes the detection of proanthocyanidins in legume seeds, the determination of anthocyanidin formation, and the strength of organoleptic astringency of partially purified proanthocyanidins.

\section{MATERIALS AND METHODS}

Raw materials. Azuki beans (Vigna angularis Ohwi et Ohashi,var. Dainagon), black soybeans (Glycine max L., Hikarikuro), soybeans (Glycine max L, an American cultivar) and mung beans (Vigna radiata Wilcz., from Indonesia) were commercially purchased.

Extraction and fractionation of proanthocyanidins. These beans were freeze-dried, powdered in a Waring blender and passed through a 12 mesh sieve. After being defatted with petroleum ether and acetone at $20^{\circ} \mathrm{C}$, each sample was dispersed in $70 \%$ aqueous acetone containing $0.1 \%$ $(\mathrm{w} / \mathrm{v})$ ascorbic acid for $20 \mathrm{hr}$ at $20^{\circ} \mathrm{C}$. The mixture was filtered and the residue was washed with $70 \%$ aqueous acetone. The filtrates and washings were combined and saturated with $\mathrm{NaCl}$ to salt out the acetone. ${ }^{5)}$ By washing the acetone phase again with water saturated with $\mathrm{NaCl}$ and acetone the crude extract was obtained. The acetone was removed in vacuo from the extract thus obtained. The residue was dissolved again in an equal volume of water. 
The aqueous solution was washed three times with diethyl ether. The aqueous phase was then extracted with an equal volume of ethyl acetate three times (Fraction-I; F-I). The aqueous phase thus obtained was then saturated with $\mathrm{NaCl}$ and reextracted with an equal volume of ethyl acetate (F-II). The residual aqueous phase was then extracted with an equal volume of acetone (F-III).

Partial purification of proanthocyanidins. Proanthocyanidins in F-I, F-II, and F-III were partially purified by Sephadex LH-20 chromatography (column size; $2.5 \times 39 \mathrm{~cm}$ ). ${ }^{6}$ After washing the Sephadex column successively with $5 \% \mathrm{EtOH}$ and $20 \% \mathrm{MeOH}$, proanthocyanidins were eluted with $60 \%$ acetone and proanthocyanidin fraction-I (PF-I), PF-II, and PF-III were obtained, respectively.

Isolation and detection of proanthocyanidins. Proanthocyanidins in PF-I were separated and detected by two-dimensional thin-layer chromatography (TLC) in the same way, as reported by Van Buren et $a l^{7)}$ Proanthocyanidin oligomers in PF-II were separated and detected by silica-gel HPTLC according to the method of Lea. ${ }^{2)}$ Proanthocyanidin polymers in PF-III were separated from each other by Sephadex $\mathrm{LH}-20$ gel filtration according to the method of Strumeyer and Malin. ${ }^{8)}$

Methods of analysis. The conversion of leucoanthocyanin to anthocyanidin, ${ }^{9}$ the controlled-hydrolysis ${ }^{10}$ ) of proanthocyanidin, the identification of acidhydrolysates $^{10)}$ and the determination of total flavans ${ }^{11 \text { ) }}$ were carried out in the same ways as described in the references.

Sensory evaluation test. The isolated proanthocyanidins were dissolved in pure water to prepare $0.0100 \%, 0.0010 \%$ and $0.0001 \%$ solutions. And $3.0 \mathrm{ml}$ aliquots of the solutions were tasted by a panel composed of five judges who were selected for their high sensitivities to organoleptic astringency.

\section{RESULTS AND DISCUSSION}

Roux $^{12)}$ reported that leucoanthocyanidins (monomeric proanthocyanidins: $\mathrm{MW}^{\prime}=290 \sim$ 322) shows no significant tanning properties. Bate-Smith ${ }^{13)}$ showed that these oligomers have tanning properties, and that the measure of relative astringency increases in the order of dimers, trimers and tetramers. Generally, the tanning action of polyphenols considerably depends on their molecular size and it was shown that the maximum tanning action, in other words, maximum astringency, is demonstrated by polyphenols of inter- mediate size (a molecular weight range of $500 \sim 3000){ }^{14)}$ Consequently, it seems important to investigate the molecular size of proanthocyanidins in order to discuss the organoleptic astringent taste of them.

Until now, however, it has been unknown whether proanthocyanidins of intermediate size are present in legume seeds or not. Therefore, at first we tried to examine the occurrence of oligomeric proanthocyanidins in some legume seeds.

It was difficult to detect oligomeric proanthocyanidins directly in the crude extracts from beans because of their lower concentrations in comparison with those of monomeric, polymeric flavan pigments, etc.
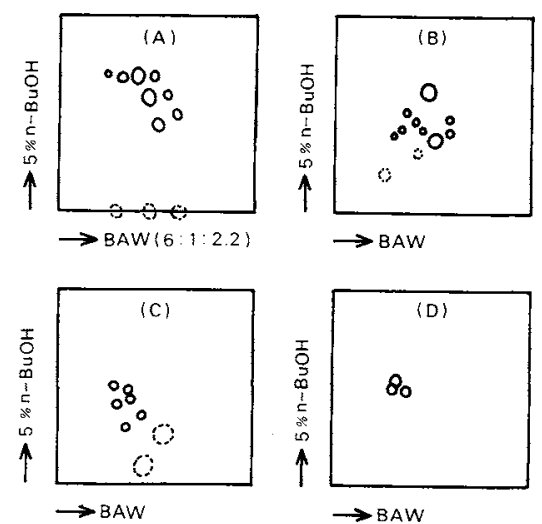

Fig. 1. Cellulose Thin-layer Chromatograms of PF-Is from Legume Seeds.

Detection reagent: vanillin-HCl. (A), azuki beans; (B), black soybeans; (C), mung beans; (D), soybeans.

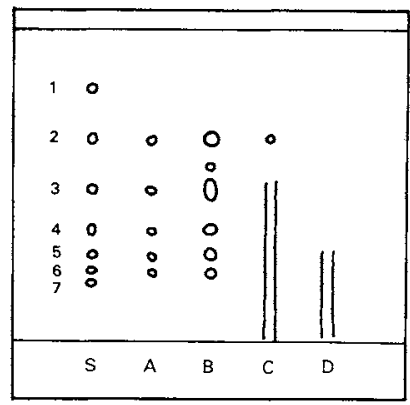

FIG. 2. Silica HPTLC of PF-IIs from Legume Seeds.

Solvent: toluene-acetone-formic acid $(3: 6: 1, \mathrm{v} / \mathrm{v} / \mathrm{v})$. Numerals indicate degree of polymerization: $S$, standards from cider $^{2 \text {; }}$ A, azuki beans; B, black soybeans; C, mung beans; D, soybeans. 


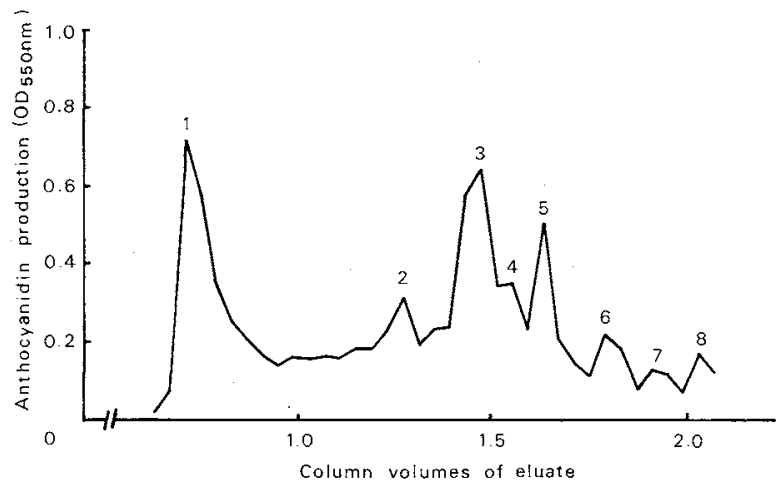

FIG. 3. Gel Filtration of PF-III from Azuki Beans on Sephadex LH-20 with $50 \%$ Aqueous Acetone.

Consequently, proanthocyanidins in the crude extract were preliminarily fractionated into three fractions, F-I, F-II, and F-III, in order of molecular weight. They were further purified by means of Sephadex LH-20 chromatography, and PF-I, PF-II, and PF-III were obtained, respectively. These PFs contained $50 \sim 88 \%$ flavans.

The HPTLC of PF-Is indicated that they were mainly composed of dimers. Since proanthocyanidins usually have many isomers, PF-Is from four kinds of beans were examined by Avicel SF cellulose TLC (Fig. 1). Actually, several spots of lower oligomers were detected for all the kind of beans. Figure 2 shows the HPTLC of PF-IIs. The results indicated the occurrence of oligomers (trimers $\sim$ hexamers) in azuki beans and black soybeans. To our knowledge, this is the first report of the occurrence of oligomeric proanthocyanidins in legume seeds. The novel technique (ethyl acetate extraction after saturating aqueous phase with $\mathrm{NaCl}$ ) was found to be very effective for the isolation of oligomers.

The HPTLC of PF-IIIs indicated that a large part of PF-IIIs is composed of polymeric flavans (proanthocyanidin type). Actually, gel filtration of PF-IIIs indicated the occurrence of many kinds of polymeric proanthocyanidins of various molecular weights in all the sorts of beans. For instance, Fig. 3 shows a gel chromatogram of PF-III from azuki beans.

The crude extract from each bean produced
TABle I. Anthocyanidin Formation $(\mathrm{mg})^{a}$

\begin{tabular}{ccccc} 
Fraction & $\begin{array}{c}\text { Azuki } \\
\text { beans }\end{array}$ & $\begin{array}{c}\text { Black } \\
\text { soybeans }\end{array}$ & $\begin{array}{c}\text { Mung } \\
\text { beans }\end{array}$ & Soybeans \\
\hline F-I & 12.0 & 5.1 & 1.1 & $>0$ \\
\hline F-II & 28.2 & 11.8 & 4.0 & $>0$ \\
\hline F-III & 78.5 & 23.6 & 59.8 & $>0.14$ \\
\hline
\end{tabular}

a Results are recorded as values per, $100 \mathrm{~g}$ of beans.

anthocyanidins $\left(\lambda_{\max }=554 \mathrm{~nm}\right.$ in $5 \% \mathrm{HCl}$ in $n$ $\mathrm{BuOH})$ on acid hydrolysis. Table I shows anthocyanidin formation ${ }^{15)}$ from . F-Is, F-IIs, and F-IIIs through acid hydrolysis. These values were supposed to indicate minimum contents of proanthocyanidins in these legume seeds. These results indicated that the oligomeric proanthocyanidin content decreases in the order of azuki beans, black soybeans, mung beans, and regular soybeans. On the other hand, the polymeric proanthocyanidin content decreases in the order of azuki beans, mung beans, black soybeans and regular soybeans. In particular, it is noteworthy that the proanthocyanidin content of black soybeans is much higher than that of regular soybeans. This result corresponds to the fact that black soybeans taste much more astringent in comparison with regular soy beans.

Treatment of each PF (PF-Is, PF-IIs, and PF-IIIs) other than that from soybeans with $0.1 \mathrm{~N}$-hydrochloric acid in ethanol at room 
temperature yielded (-)epi-catechin and unknown products on the paper chromatogram. Treatment with $5 \mathrm{~N}$-hydrochloric acid in ethanol at $60^{\circ} \mathrm{C}$ gave cyanidin and unknown products. As for the PFs from soybeans, these phenomena could not be confirmed because of the shortage of samples due to the low content of these substances.

Aqueous solutions of each PF other than that from soybeans tasted astringent even at a concentration of $0.0010 \%(w / v)$. As for the organoleptic taste of PFs from soybeans, it was impossible to evaluate it for the same reason mentioned above. These results suggested that these PFs may taste more astringent than any other natural compounds reported. ${ }^{16)}$

PF-IIIs as well as PF-Is and PF-IIs showed significant organoleptic astringency. However, aqueous solutions of highly polymeric proanthocyanidins such as peak-1 and peak-2 (Fig. 3), which contained no proteinaceous constituents, did not taste astringent at the concentration of $0.0100 \%$. This result suggests that the PF-III from azuki beans contains both non-astringent proanthocyanidins (higher polymers) and astringent proanthocyanidins (lower polymers).

In conclusion, oligomeric and low polymeric proanthocyanidins may perhaps contribute to the astringent off-taste of legume seeds such as azuki beans, black soybeans, and mung beans.
Acknowledgments. The authors wish to express their thanks to Professor M. Fujimaki of Ochanomizu University, and Professor H. Kato, Professor S. Arai and Dr. M. Yamashita of the University of Tokyo for their valuable suggestions and encouragement in this work. Thanks are also due to Dr. N. Iguchi and Dr. F. Yoshida of these laboratories for their encouragement and to Dr. I. Koshiyama of the same organization for his kind advice.

\section{REFERENCES}

1) T. A. Geissman and H. F. K. Dittmar, Phytochemistry, 4, 359 (1965).

2) A. G. H. Lea, J. Sci. Food Agric., 29, 471 (1978).

3) J. Martin-Tanguy, J. Guillaume and A. Kossa, J. Sci. Food Agric., 28, 757 (1977).

4) M. L. Price, A. E. Hagerman and L. G. Butler, $J$. Agric. Food Chem., 28, 459 (1980).

5) W. T. Jones, R. B. Broadhurst and J. W. Lyttleton, Phytochemistry, 15, 1407 (1976).

6) A. G. H. Lea and C. F. Timberlake, J. Sci. Fond Agric., 25, 1537 (1974).

7) J. P. Van Buren, G. Senn and H. Neukom, J. Food Sci., 31, 964 (1966).

8) D. H. Strumeyer and M. J. Malin, J. Agric. Food Chem., 23, 909 (1975).

9) H. E. Hillis, J. Sci. Food Agric., 10, 63 (1959).

10) R. S. Thompson, D. Jacques, E. Haslam and R. J. N. Tanner, J. Chem. Soc. Perkin I, 11, 1387 (1972).

11) R. B. Broadhurst and W. T. Jones, J. Sci. Food Agric., 29, 788 (1978).

12) D. G. Roux, Phytochemistry, 11, 1219 (1972).

13) E. C. Bate-Smith, Phytochemistry, 12, 907 (1973).

14) E. Haslam, "The Flavonoids," ed. by J. B. Harbone et al., Chapman and Hall Ltd., London, 1975, p. 528.

15) E. C. Bate-Smith, Phytochemistry, 14, 1107 (1975).

16) M. Nakagawa, Nippon Shokuhin Kogyo Gakkaishi, 19, 531 (1972). 